\title{
Controversy Surrounding the Function of SpiC Protein in Salmonella: An Overview
}

\section{Yaonan Wang ${ }^{1}$, Yuan Cai', Jian Zhang ${ }^{1}$, Dong Liu' ${ }^{2}$ Xiao Gong ${ }^{2}$, Zhiming Pan', Shizhong Geng ${ }^{1 *}$ and Xin'an Jiao ${ }^{1}$}

${ }^{1}$ College of Bioscience and Biotechnology and College of Veterinary Medicine, Jiangsu Key Laboratory of Zoonosis, Jiangsu Co-Innovation Center for Prevention and Control of Important Animal Infectious Diseases and Zoonoses, Yangzhou University, Yangzhou, China, ${ }^{2}$ Research and Development Center, State Key Laboratory of Genetically Engineered Veterinary Vaccines, Yebio Bioengineering Co., Ltd of Qingdao, Qingdao, China

Salmonella is an important pathogenic microorganism that can infect humans and animals and has been studied globally as a model microorganism for its pathogenesis. The SpiC protein of T3SS2 is a significant factor that has been studied for almost 20 years, but to date, the function/effect of SpiC in the pathogenesis of Salmonella has not been completely understood. There is controversy over the functions of SpiC protein in the literature. Thus, an overview of the literature on SpiC protein is provided here which highlights expression features of SpiC protein and its various functions and effect.

Keywords: salmonella, T3SS2, SpiC protein, virulence factor, pathogenesis

\section{INTRODUCTION}

Salmonella spp. is an important food-borne zoonotic pathogen, belonging to the family Enterobacteriaceae, which infects millions of people and livestock, even leading to human and animal death causing severe economic losses every year (Taylor et al., 2018). It had two species including Salmonella Bongori and Salmonella enterica and six subspecies in the specie Salmonella enterica (Gal-Mora, 2019), over 2,600 serovars have been found (Geng et al., 2016).

Twenty-three Salmonella pathogenicity islands (SPIs) have been identified to be involved in Salmonella pathogenic mechanism (Fookes et al., 2011; Hayward et al., 2013). Among them, SPI1 and SPI2 are two major SPIs, encoding virulence-related type III secretion system (T3SS), T3SS1 and T3SS2, respectively. Proteins secreted by these two SPIs are involved in pathogenicity at the molecular level and provide a novel insight into Salmonella pathogenesis. T3SS2 is not found in Salmonella Bongori, but in six subspecies of Salmonella enterica. Low $\mathrm{pH}$ and nutrient shortage in the Salmonella-containing vacuole (SCV) can induce intracellular expression of the T3SS2 (Rappl et al., 2003; Allam et al., 2012). These secreted effectors can modulate the intracellular environment (Deng et al., 2017). During Salmonella infection, a variety of virulence factors and effectors are expressed to be translocated across the membrane of the SCV to cytoplasm of infected host cell by the T3SS2 and interact with proteins in host cell to antagonize innate immune defense of host cell, supporting its survival and replication for persistent infection (Schleker et al., 2015). 
SpiC was the first SPI2-encoded protein to be identified that could be secreted by the Spi/Ssa T3SS2 system into the macrophage cytoplasm (Ochman et al., 1996; Uchiya et al., 1999). A spiC-deletion mutant had significantly reduced virulence in mice. However, the function of the SpiC protein remains unclear and is controversial (Kujat Choy et al., 2004; Figueira and Holden, 2012). It is, therefore, necessary to summarize recent works on the function of $\mathrm{SpiC}$ as a reference for future studies. The controversies are presented as follows:

\section{The Size of SpiC Protein: 133 aa or 127 aa}

SpiC, as a virulence factor, is species-specific, comparing with disease pathology between Salmonella and Shigella. This protein has two isoforms: 133 amino acid (aa) or 127 aa, depending on the translational start site. Presumably, the 127-amino acid ORF is translated form "AGGAG," the sequence of its putative ribosome-binding site at the $3^{\prime}$ end of the $16 \mathrm{~S}$ rRNA (Ochman et al., 1996). In the first published paper, the 127 aa isoform was studied (Ochman et al., 1996). As an effector protein, SpiC was suggested to be expressed by SPI2 (Ochman et al., 1996; Hensel et al., 1998; Uchiya et al., 1999) without the conserved N-terminal amino acids, which exists in most of translocated effectors that have been confirmed (Miao and Miller, 2000).

But in many studies, the 133 aa isoform was studied. For example, the level of intramacrophage expression of the spiC gene with $402 \mathrm{bp}$ (133 aa) is 52.99 -fold greater than that of the ESP (ESP refers to bacterial growth in Lennox broth to $\left.\mathrm{OD}_{600}=2.0\right)$ (Srikumar et al., 2015).

An adenine is the transcription start site of $s p i C$, at 18 basepases upstream of initiation codon (ATG) of the spiC gene, which is consistent with the finding of Walthers et al. (Walthers et al., 2007). SpiC protein contains 127 amino acids, with a molecular weight of approximately $14.7 \mathrm{kDa}$.

\section{Which One Controls SpiC Expression: T3SS1 or T3SS2?}

SpiC is expressed during all stages of infection by T3SS2 and spiC gene usually represents T3SS2 in the literature (Bruce et al., 2018); however, SpiC/SsaB is secreted in the early stages of SCV biogenesis (<30 min) by T3SS1 (Steele-Mortimer, 2008).

\section{Where Do Salmonella Express SpiC: Inside or Outside of Infected Cells? Intracellular Expression Inside of Infected Cells}

SpiC protein expressed in Salmonella might be a regulator of, or chaperone for, the translocon protein SseBC (Freeman et al., 2002); SpiC may control the expression of flagella and fliC since their expression level was lower in Salmonella spiC-deleted mutant than that in parent Salmonella (Uchiya and Nikai, 2009). SpiC acts as a "gatekeeper" enabling translocon proteins, such as SseBC and SseJ, to be secreted from Salmonella (Büttner, 2012). SpiC-2HA, a tagged protein, could only been found in bacteria, not in the cytoplasm and membrane of infected cells (Yu et al., 2002, 2004). Salmonella in the SCV do not secrete SpiC into the cytoplasm.
After extracelluar growth of Salmonella spiC-deleted mutant and its complementary strain at low $\mathrm{pH}$ to induce SPI2-encoded protein secretion. The SpiC protein cannot be detected in Salmonella culture media or on the tube inner surface (Shea et al., 1999; Yu et al., 2002). These results are consistent with the research result of Hansen-Wester (Hansen-Wester et al., 2002), a SpiC::M45 tagged protein cannot be detected under similar culture conditions (Yu et al., 2002).

SpiC protein might be a non-effector of the SPI2 T3SS because of failed attempts to show that SpiC was a translocated protein. It might be one kind of SPI2-encoded translocons, but not an effector (Freeman et al., 2002; Yu et al., 2002).

When a host cell is attacked, Salmonella Typhimurium expresses a higher level of spiC mRNA than Salmonella Infantis to survive within macrophages (Braukmann et al., 2015).

Extracellular Expression Outside of Infected Cells In addition to being a member of the regulatory complex, SpiC is secreted by T3SS2 into the eukaryotic cytosol to interfere with cellular behavior (Ramos-Morales, 2012). SpiC can be translocated into the macrophage cytoplasm (Uchiya et al., 1999). It can also act with Hook3 and TassC in the cytosol to interfere with vesicle trafficking to block SCV from fusing with lysosomes and incoming endosomes (Kuhle and Hensel, 2004; Valdez et al., 2009). Thus, Salmonella in the SCV can secrete SpiC into the cytosol. Salmonella-containing vacuoles normally fuse with lysosomes within $20 \mathrm{~min}$ of infection. SpiC may have profound effects on the host cell to block this fusion. SpiC cannot be detected until approximately $1 \mathrm{~h}$ after infection. SpiC production may begin while the bacteria are in other cells, but before they enter macrophages, because macrophages rely on vesicle fusion to secrete factors that stimulate and attract other cells of the immune system (Strauss, 1999).

In addition, PspiC (promoter of spiC) and PssaG (promoter of $s s a G$ ) strains were constructed by promoter fusion for RIVET analysis of $s p i C$ transcription. Typical SPI2 expression features were proved during in vitro culture. After PspiC and PssaG strains were orally infected into mouse ileal loops, SpiC protein could also be detected at 15 min post infection (Brown et al., 2005). These results show that SpiC protein can be expressed extracellularly in a normal culture.

Wild-type Salmonella harbouring a transcriptional fusion of the spiC promoter to a promoter-less $g f p$ gene in plasmid pFPV25 showed high levels of spiC expression $6 \mathrm{~h}$ after infection. This resulted in an 11-fold induction of intramacrophages spiC expression when compared with that in tissue culture media (Bijlsma and Groisman, 2005). A spiC::lacZ fusion transcription could be detected in in vitro culture of Salmonella in low-osmolarity N salt medium (Deiwick et al., 1999). Fimbriae with an integrated viral epitope can be induced to expression from the spiC promoter in LB containing different concentrations of $\mathrm{Mg}^{2+}$ in a cya-crp-pgtE Salmonella mutant (Chen and Schifferli, 2003).

Salmonella Paratyphi A 45157 and Salmonella Typhimurium SL1344 were cultured to the stationary phase in vitro at 37 or $42^{\circ} \mathrm{C}$ under microaerophilic conditions and harvested for 
total RNA, which was performed by qpCR, mRNA level of spiC gene was increased by 30 -fold at $42^{\circ} \mathrm{C}$ compared with that at $37^{\circ} \mathrm{C}$ (Elhadad et al., 2015).

Organelles such as the SCV, lysosomes (Hashim et al., 2000), phagosomes (Buchmeier and Heffron, 1991), and Golgi body (Shotland et al., 2003) and proteins such as transferrin (Uchiya et al., 1999; Pan et al., 2010), Rab (Buchmeier and Heffron, 1991), HooK3 (Shotland et al., 2003), TassC and NIPSNAP (NSP4) (Lee et al., 2002) interact in the cell. SpiC protein from Salmonella can interact with Hook3 protein to affect cellular trafficking (Shotland et al., 2003; Valdez et al., 2009), SpiC protein could enter into the cytoplasm of Salmonella-infected cells.

\section{SpiC Invovle in Virulence: Virulence or Non-virulence Proteins \\ Virulence Protein}

The $\mathrm{LD}_{50}$ of the spiC-inactivated Salmonella Pullorum S06004 was $1.2 \times 10^{9} \mathrm{CFU}$, and up to 200 -fold greater compared with that of the wild-type S06004 $\left(6.0 \times 10^{6} \mathrm{CFU}\right)$ (Geng et al., 2014). Similar results were obtained for Salmonella Gallinarum (Cheng et al., 2016).

The $\operatorname{LD}_{50}\left(2.87 \times 10^{6} \mathrm{CFU}\right)$ of a spiC-deleted Salmonella enteritidis C50041 was 900-fold higher than that of the wildtype strain $\left(3.11 \times 10^{3} \mathrm{CFU}\right)$ when chickens were infected (Zeng et al., 2015). Another research proposed that the spiCdeleted mutant of Salmonella Typhimurium was of low virulence in intraperitoneally inoculated mice. The $\mathrm{LD}_{50}$ of the spiC-deleted mutant was over $3.63 \times 10^{6} \mathrm{CFU}$, but the LD50 of wild-type Salmonella Typhimurium was below $10 \mathrm{CFU}$ (Uchiya et al., 1999).

Intracellular survival and replication are important virulence determinants (Ibarra and Steele-Mortimer, 2009). The intramacrophage growth of Salmonella enteritidis C50041 with a spiC-deletion was continuous, while the number of wildtype Salmonella began to reduce from 10 to $24 \mathrm{~h}$ post infection, but the load of spiC-deleted mutants began to increase, which was significantly higher compared with that of the wild-type Salmonella in RAW264.7 macrophages at $24 \mathrm{~h}$ post infection (Data by our Lab).

Statistical analysis of Grant's research showed the load of Salmonella SPI2 mutants was higher per cell than that of wildtype Salmonella. The ssaV, ssaM, spiC, and sseB mutants can survive in far fewer infected cells per organ, with higher loads per cell than that of the wild type (Grant et al., 2012). SpiC was necessary for Salmonella Typhimurium spread in the spleen (Grant et al., 2012). The intracellular spiC-deleted mutant was suggested to change the metabolic pathway for its proliferation using NO (Nitric oxide) as a nitrogen source (Das et al., 2009).

However, Uchiya's research results showed that the spiC-deleted mutant of Salmonella Typhimurium could not survive within macrophages (Uchiya et al., 1999). The intramacrophage survival of the spiC-deleted mutant of Salmonella Typhimurium 14028s was less than $20 \%$ compared with $100 \%$ of wild-type Salmonella Typhimurium 14028s in $\mathrm{J774}$ macrophages at $18 \mathrm{~h}$ post infection (Uchiya et al., 1999). In the typhoid model, Buckner et al. found that $\triangle$ spiC mutant was attenuated for colonization of intestinal and systemic sites. The $\Delta$ spiC mutant replicated to the same extent as the wild type in epithelial cells but replicated to a poorer extent in macrophages (Buckner et al., 2011).

\section{Non-virulence Protein}

In an unpublished report by Ajay Singh, the function of the SpiC protein was also analyzed in Caenorhabditis elegans, another model of Salmonella Typhimurium infection. The conclusions showed that the loss of spiC did not change the virulence of Salmonella Typhimurium and the lethality of infection in Caenorhabditis elegans because of the similar rate of death with the wild-type Salmonella group.

\section{Where SpiC Function/Effect: In Salmonella or the Host Cells In Salmonella}

A spiC-deleted mutant of Salmonella Typhimurium could not secrete SPI2 proteins again (Yu et al., 2002). After host cells are infected by Salmonella, the bacteria can survive in the SCV in the cell and transfer the translocon protein SseBC; the secretion of these effectors is regulated by SpiC protein (Freeman et al., 2002) through the needle of the T3SS to link the SCV membrane. Theoretically, translocon proteins must be expressed and secreted to guide effectors to be translocated. In an in vitro assay, SpiC was one component of SsaL/SsaM/SpiC complexes to act as a "gatekeeper" to enable translocon and effector protein secretion at $\mathrm{pH}$ 5.0, when wild-type Salmonella was exposed to $\mathrm{pH} 7.2$ of host cell cytoplasm by the translocon pore and the canal of the needle at $\mathrm{pH} 5.0$ and dissociation and degradation of SsaM/SpiC/SsaL complexes are brought about (Rappl et al., 2003; Yu et al., 2010). SpiC was therefore deemed to be a switch regulator (Walthers et al., 2007).

The SPI2 effector SseJ and PipB are oversecreted in in vitro ssaM and spiC-deleted mutants. Immunoblot analysis revealed that at most $5 \%$ of the total SpiC protein could be translocated into host cell cytoplasm (Yu et al., 2004). SpiC protein participated in the synthesis of FliC, which could activate SPI2-dependent MAPK pathways in macrophages infected by Salmonella (Uchiya and Nikai, 2008).

There were many flagella filaments on the wild-type Salmonella surface, while only a few flagella could be observed on the spiC-deleted mutant surface. SpiC participated in flagellum assembly by changing flhDC gene expression, which is a primary regulator of flagella synthesis and assembly. The spiC-deleted mutant of Salmonella Typhimurium is defective in flagella filament formation and shows a severe defect in motility (Uchiya and Nikai, 2009; Valdez et al., 2009). However, our results showed no change in flagella and swim/swarm ability between the spiC-deleted mutant and wild-type Salmonella enteritidis C50041 (Valdez et al., 2009).

\section{In Cells}

SpiC can interact with its target proteins in host cells but it does not serve as an independent toxin-like effector (Lee et al., 2002). An intracellular hook protein and the 
NIPSNAP homolog was confirmed to be a target of SpiC protein (Shotland et al., 2003).

SpiC protein blocks cellular traffic (just as one crucial accident can slow activity throughout a city) and interrupts Salmonellacontaining vesicle fusion with lysosomes. In general, macrophages rely on vesicle fusion (Salmonella-containing vesicles fuse with lysosomes) (Hashim et al., 2000) to secrete factors that stimulate and attract other cells of the immune system. SpiC-mediated blockage of vesicle fusion might also affect macrophage activity in unanticipated ways and hamper immune system functioning (Strauss, 1999). This disruption protects pathogens by antagonizing against cell's bactericidal contents, such as reactive oxygen and reactive nitrogen species (Kaur and Jain, 2012).

Compared with the spiC-deleted Salmonella, prostaglandin E2 (PGE2) and prostacyclin (PGI2) were highly expressed and activated the PKA signaling pathway by corresponding receptors and the phosphorylation of ERK1/2 was higher level in J774 macrophages infected by parent Salmonella (Uchiya and Nikai, 2004). MAPK pathways in macrophages infected by Salmonella could be activated SPI2-dependently, SpiC as major effector regulates the expression of $\mathrm{FliC}$, which is involved in MAPK pathways (Uchiya and Nikai, 2008; Valdez et al., 2009). JAK/STAT signaling pathway could be inhibited by cytokine signaling 3 (SOCS-3), SOCS-3 was highly expressed in J774 macrophages infected by parent Salmonella compared with that by spiC-deleted mutant. Salmonella causing spiC-dependent ERK1/2 activation and then leading to SOCS-3 expression (Uchiya and Nikai, 2005; Valdez et al., 2009). Interleukin-10 (IL-10) mRNA was highly expressed in macrophages infected by parent Salmonella compared with that by spiC-deleted mutant (Uchiya et al., 2004). Cyclic AMP-dependent PKA showed higher activity in macrophages infected by parent Salmonella compared with that by spiC-deleted mutant, but there were no obvious difference of the levels of IL-1 $\beta$, IL-6, and TNFa (Uchiya and Nikai, 2004).

The assembly of F-actin protein was regulated by SpiC in intracellular Salmonella Typhimurium (Holden, 2002; Yu et al., 2002). A spiC-deleted mutant cannot form SCV filaments, suggesting that another SPI2 effector SifA may not translocate in this spiC-deleted mutant (Lee et al., 2002; Valdez et al., 2009). spiC-deleted ST240 can not produce completely generate lgp (lysosomal membrane glycoprotein)-tubules within HeLa cells (Guy et al., 2000). SIF formation is controlled by SpiC, SseF, and SseG (Kuhle and Hensel, 2004). SIF formation and translocons secretion in the spiC complementary Salmonella based on plasmid-expression was restored (Yu et al., 2004).

\section{Other Functions/Effect}

SPI2 mutant strains were attenuated in vivo, showing reduced tissue colonization and enhanced T-cell activation, which confers protection against a challenge with wild-type virulent Salmonella (Tobar et al., 2006). SpiC was also suggested to inhibit dendritic cell movement and antigen delivery (McLaughlin et al., 2014), thus inhibiting Salmonella from escaping the infected cell to spread throughout the body and mediating Salmonella to antagonize (resist) host cell defenses, including respiratory burst $\left(\mathrm{NO}, \mathrm{H}_{2} \mathrm{O}_{2}\right.$, $\mathrm{NADPH}$ ), the inflammatory response, and cellular autophagy (Vazquez-Torres et al., 2000; McCollister et al., 2005; Bourret et al., 2009; Das et al., 2009). The spiC-deleted mutant complementated with a plasmid containing spiC allele restored the P-body (dynamic aggregates of RNA and proteins) disassembly phenotype (Eulalio et al., 2011).

In our laboratory, the spiC mutant of Salmonella Pullorum which was highly attenuated was found to persist in spleen and liver for less than 10 days and induced high levels of circulating antibody and protective immunity against oral challenge in young broiler chickens. It showed that the spiC mutant is a potential new vaccine candidate for use with chickens against this disease (Geng et al., 2014). Then $1009 \Delta$ spiC $\Delta$ crp, a spiC and crp deletion mutant of Salmonella Gallinarum, was evaluated in chickens. $1009 \Delta$ spiC $\Delta$ crp bacteria colonized and persisted in the liver and spleen of vaccinated chickens for $>14$ days, and significant specific humoral and cellular immune responses were induced. Efficient protection was observed after vaccinated chickens were challenged with Salmonella Gallinarum SG9 at 21 days post-immunization. These results demonstrate that $1009 \Delta$ spiC $\Delta c r p$ can be used as a live attenuated vaccine (Cheng et al., 2016). In addition, Salmonella Pullorum $\Delta$ spiC $\Delta$ waaL was found to facilitate the differentiation between infected and vaccinated chickens. What is more, the vaccine candidate showed adequate safety being avirulent in the host chicks. Single intramuscular immunization of day-old broiler chicks with the mutant confers ideal protection against lethal wild-type challenge by significantly stimulating both humoral and cellular immune responses as well as reducing the colonization of the challenge strain. The mutant strain generated cross-protection against challenge with the wild-type Salmonella Gallinarum. These results suggest that the doublemutant strain may be a safe, effective, and cross-protective vaccine against Salmonella infection in chicks while conforming to the requirements of the DIVA program (Guo et al., 2017).

However, the previous studies were all related to vaccine research and development. The underlying mechanisms remain unclear, which is needed to be studied in the future.

\section{AUTHOR CONTRIBUTIONS}

YW, YC, and JZ collected all references. YW and SG drafted the manuscript. DL, XG, ZP, and XJ revised and finalized the manuscript. All authors read and approved the final version of the manuscript.

\section{FUNDING}

This work was supported by the National Key Research and Development Program Special Project (2016YFD0501607), the Special Fund for Agroscientific Research in the Public Interest (201403054), the Natural Science Foundation of Jiangsu Province of China (BK20151306), the Yangzhou University Science and Technology Innovation Team, and the Priority Academic Program Development of Jiangsu Higher Education Institutions (PAPD), and Open Project of State Key Laboratory of Genetically Engineered Veterinary Vaccines (AGVSKL-ZY-201803). 


\section{REFERENCES}

Allam, U. S., Krishna, M. G., Sen, M., Thomas, R., Lahiri, A., Gnanadhas, D. P., et al. (2012). Acidic pH induced STM1485 gene is essential for intracellular replication of Salmonella. Virulence 3, 122-135. doi: 10.4161/viru.19029

Bijlsma, J. J., and Groisman, E. A. (2005). The PhoP/PhoQ system controls the intramacrophage type three secretion system of Salmonella enterica. Mol. Microbiol. 57, 85-96. doi: 10.1111/j.1365-2958.2005.04668.x

Bourret, T. J., Song, M., and Vazquez-Torres, A. (2009). Codependent and independent effects of nitric oxide-mediated suppression of PhoPQ and Salmonella pathogenicity island 2 on intracellular Salmonella enterica serovar Typhimurium survival. Infect. Immun. 77, 5107-5115. doi: 10.1128/IAI.00759-09

Braukmann, M., Methner, U., and Berndt, A. (2015). Immune reaction and survivability of Salmonella Typhimurium and Salmonella Infantis after infection of primary avian macrophages. PLoS One 10:e0122540. doi: 10.1371/journal. pone. 0122540

Brown, N. F., Vallance, B. A., Coombes, B. K., Valdez, Y., Coburn, B. A., and Finlay, B. B. (2005). Salmonella pathogenicity island 2 is expressed prior to penetrating the intestine. PLoS Pathog. 1:e32. doi: 10.1371/journal. ppat.0010032

Bruce, H. L., Barrow, P. A., and Rycroft, A. N. (2018). Zoonotic potential of Salmonella enterica carried by pet tortoises. Vet. Rec. 182, 141-144. doi: 10.1136/vr.104457

Buchmeier, N. A., and Heffron, F. (1991). Inhibition of phagosome-lysosome fusion by Salmonella Typhimurium. Infect. Immun. 59, 2232-2238.

Buckner, M. M., Croxen, M. A., Arena, E. T., and Finlay, B. B. (2011). A comprehensive study of the contribution of Salmonella enterica serovar Typhimurium SPI2 effectors to bacterial colonization, survival, and replication in typhoid fever, macrophage, and epithelial cell infection models. Virulence 2, 208-216. doi: 10.4161/viru.2.3.15894

Büttner, D. (2012). Protein export according to schedule: architecture, assembly, and regulation of type III secretion systems from plant- and animal-pathogenic bacteria. Microbiol. Mol. Biol. Rev. 76, 262-310. doi: 10.1128/MMBR.05017-11

Chen, H., and Schifferli, D. M. (2003). Construction, characterization, and immunogenicity of an attenuated Salmonella enterica serovar typhimurium $p g t E$ vaccine expressing fimbriae with integrated viral epitopes from the spiC promoter. Infect. Immun. 71, 4664-4673. doi: 10.1128/IAI.71.8.4664-4673.2003

Cheng, Z., Yin, J., Kang, X., Geng, S., Hu, M., Pan, Z., et al. (2016). Safety and protective efficacy of a spiC and crp deletion mutant of Salmonella gallinarum as a live attenuated vaccine for fowl typhoid. Res. Vet. Sci. 107, 50-54. doi: 10.1016/j.rvsc.2016.05.007

Das, P., Lahiri, A., Lahiri, A., and Chakravortty, D. (2009). Novel role of the nitrite transporter NirC in Salmonella pathogenesis: SPI2-dependent suppression of inducible nitric oxide synthase in activated macrophages. Microbiology 155, 2476-2489. doi: 10.1099/mic.0.029611-0

Deiwick, J., Nikolaus, T., Erdogan, S., and Hensel, M. (1999). Environmental regulation of Salmonella pathogenicity island 2 gene expression. Mol. Microbiol. 31, 1759-1773. doi: 10.1046/j.1365-2958.1999.01312.x

Deng, W., Marshall, N. C., Rowland, J. L., McCoy, J. M., Worrall, L. J., Santos, A. S., et al. (2017). Assembly, structure, function and regulation of type III secretion systems. Nat. Rev. Microbiol. 15, 323-337. doi: 10.1038/ nrmicro.2017.20

Elhadad, D., McClelland, M., Rahav, G., and Gal-Mor, O. (2015). Feverlike temperature is a virulence regulatory cue controlling the motility and host cell entry of Typhoidal Salmonella. J. Infect. Dis. 212, 147-156. doi: 10.1093/ infdis/jiu663

Eulalio, A., Frohlich, K. S., Mano, M., Giacca, M., and Vogel, J. (2011). A candidate approach implicates the secreted Salmonella effector protein SpvB in P-body disassembly. PLoS One 6:e17296. doi: 10.1371/journal.pone.0017296

Figueira, R., and Holden, D. W. (2012). Functions of the Salmonella pathogenicity island 2 (SPI2) type III secretion system effectors. Microbiology 158, 1147-1161. doi: 10.1099/mic.0.058115-0

Fookes, M., Schroeder, G. N., Langridge, G. C., Blondel, C. J., Mammina, C., Connor, T. R., et al. (2011). Salmonella bongori provides insights into the evolution of the Salmonellae. PLoS Pathog. 7:e1002191. doi: 10.1371/journal. ppat.1002191

Freeman, J. A., Rappl, C., Kuhle, V., Hensel, M., and Miller, S. I. (2002). SpiC is required for translocation of Salmonella pathogenicity island 2 effectors and secretion of translocon proteins SseB and SseC. J. Bacteriol. 184, 4971-4980. doi: 10.1128/JB.184.18.4971-4980.2002

Gal-Mora, O. (2019). Persistent infection and long-term carriage of typhoidal and nontyphoidal Salmonellae. Clin. Microbiol. Rev. 32:e00088-18. doi: 10.1128/ CMR.00088-18

Geng, S., Jiao, X., Barrow, P., Pan, Z., and Chen, X. (2014). Virulence determinants of Salmonella Gallinarum biovar Pullorum identified by PCR signaturetagged mutagenesis and the spiC mutant as a candidate live attenuated vaccine. Vet. Microbiol. 168, 388-394. doi: 10.1016/j.vetmic.2013.11.024

Geng, S., Tian, Q., An, S., Pan, Z., Chen, X., and Jiao, X. (2016). High-efficiency, two-step scarless-markerless genome genetic modification in Salmonella enterica. Curr. Microbiol. 72, 1-7. doi: 10.1007/s00284-016-1002-3

Grant, A. J., Morgan, F. J. E., McKinley, T. J., Foster, G. L., Maskell, D. J., and Mastroeni, P. (2012). Attenuated Salmonella Typhimurium lacking the pathogenicity island-2 type 3 secretion system grow to high bacterial numbers inside phagocytes in mice. PLoS Pathog. 8:e1003070. doi: 10.1371/journal.ppat.1003070

Guo, R., Jiao, Y., Li, Z., Zhu, S., Fei, X., Geng, S., et al. (2017). Safety, protective immunity, and DIVA capability of a rough mutant Salmonella pullorum vaccine candidate in broilers. Front. Microbiol. 8:547. doi: 10.3389/fmicb.2017.00547

Guy, R. L., Gonias, L. A., and Stein, M. A. (2000). Aggregation of host endosomes by Salmonella requires SPI2 translocation of SseFG and involves SpvR and the fms-aroE intragenic region. Mol. Microbiol. 37, 1417-1435. doi: 10.1046/j. 1365-2958.2000.02092.x

Hansen-Wester, I., Stecher, B., and Hensel, M. (2002). Type III secretion of Salmonella enterica serovar Typhimurium translocated effectors and SseFG. Infect. Immun. 70, 1403-1409. doi: 10.1128/IAI.70.3.1403-1409.2002

Hashim, S., Mukherjee, K., Raje, M., Basu, S. K., and Mukhopadhyay, A. (2000). Live Salmonella modulate expression of Rab proteins to persist in a specialized compartment and escape transport to lysosomes. J. Biol. Chem. 275, 16281-16288. doi: 10.1074/jbc.275.21.16281

Hayward, M. R., Jansen, V. A., and Woodward, M. J. (2013). Comparative genomics of Salmonella enterica serovars Derby and Mbandaka, two prevalent serovars associated with different livestock species in the UK. BMC Genomics 14, 1-19. doi: 10.1186/1471-2164-14-365

Hensel, M., Shea, J. E., Waterman, S. R., Mundy, R., Nikolaus, T., Banks, G., et al. (1998). Genes encoding putative effector proteins of the type III secretion system of Salmonella pathogenicity island 2 are required for bacteria virulence and proliferation in macrophages. Mol. Microbiol. 30, 163-174. doi: 10.1046/j.1365-2958.1998.01047.x

Holden, D. W. (2002). Trafficking of the Salmonella vacuole in macrophages. Traffic 3, 161-169. doi: 10.1034/j.1600-0854.2002.030301.x

Ibarra, J. A., and Steele-Mortimer, O. (2009). Salmonella-the ultimate insider. Salmonella virulence factors that modulate intracellular survival. Cell. Microbiol. 11, 1579-1586. doi: 10.1111/j.1462-5822.2009.01368.x

Kaur, J., and Jain, S. K. (2012). Role of antigens and virulence factors of Salmonella enterica serovar Typhi in its pathogenesis. Microbiol. Res. 167, 199-210. doi: 10.1016/j.micres.2011.08.001

Kuhle, V., and Hensel, M. (2004). Cellular microbiology of intracellular Salmonella enterica: functions of the type III secretion system encoded by Salmonella pathogenicity island 2. Cell. Mol. Life Sci. 61, 2812-2826. doi: 10.1007/ s00018-004-4248-z

Kujat Choy, S. L., Boyle, E. C., Gal-Mor, O., Goode, D. L., Valdez, Y., Vallance, B. A., et al. (2004). SseK1 and SseK2 are novel translocated proteins of Salmonella enterica serovar Typhimurium. Infect. Immun. 72, 5115-5125. doi: 10.1128/IAI.72.9.5115-5125.2004

Lee, A. H., Zareei, M. P., and Daefler, S. (2002). Identification of a NIPSNAP homologue as host cell target for Salmonella virulence protein SpiC. Cell. Microbiol. 4, 739-750. doi: 10.1046/j.1462-5822.2002.00225.x

McCollister, B. D., Bourret, T., Gill, R., Jones-Carson, J., and Vázquez-Torres, A. (2005). Repression of SPI2 transcription by nitric oxide-producing, IFN $\gamma$ activated macrophages promotes maturation of Salmonella phagosomes. J. Exp. Med. 202, 625-635. doi: 10.1084/jem.20050246

McLaughlin, L. M., Xu, H., Carden, S. E., Fisher, S., Reyes, M., Heilshorn, S. C., et al. (2014). A microfluidic-based genetic screen to identify microbial virulence factors that inhibit dendritic cell migration. Integr. Biol. 6, 438-449. doi: 10.1039/C3IB40177D

Miao, E. A., and Miller, S. I. (2000). A conserved amino acid sequence directing intracellular type III secretion by Salmonella typhimurium. PNAS 97, 7539-7544 doi: $10.1073 /$ pnas.97.13.7539 
Ochman, H., Soncini, F. C., Solomon, F., and Groisman, E. A. (1996). Identification of a pathogenicity island required for Salmonella survival in host cells. Proc. Natl. Acad. Sci. USA 93, 7800-7804.

Pan, X., Tamilselvam, B., Hansen, E. J., and Daefler, S. (2010). Modulation of iron homeostasis in macrophages by bacterial intracellular pathogens. BMC Microbiol. 10:13. doi: 10.1186/1471-2180-10-64

Ramos-Morales, F. (2012). Impact of Salmonella enterica type III secretion system effectors on the eukaryotic host cell. ISRN Cell Biol. 2012, 1-36. doi: $10.5402 / 2012 / 787934$

Rappl, C., Deiwick, J., and Hensel, H. (2003). Acidic pH is required for the functional assembly of the type III secretion system encoded by Salmonella pathogenicity island 2. FEMS Microbiol. Lett. 226, 363-372. doi: 10.1016/ S0378-1097(03)00638-4

Schleker, S., Kshirsagar, M., and Klein-Seetharaman, J. (2015). Comparing human-Salmonella with plant-Salmonella protein-protein interaction predictions. Front. Microbiol. 6:45. doi: 10.3389/fmicb.2015.00045

Shea, J. E., Beuzon, C. R., Gleeson, C., Mundy, R., and Holden, D. W. (1999). Influence of the Salmonella typhimurium pathogenicity island type III secretion system on bacterial growth in the mouse. Infect. Immun. 67, 213-219.

Shotland, Y., Kramer, H., and Groisman, E. A. (2003). The Salmonella SpiC protein targets the mammalian Hook3 protein function to alter cellular trafficking. Mol. Microbiol. 49, 1565-1576. doi: 10.1046/j.1365-2958.2003.03668.x

Srikumar, S., Kröger, C., Hébrard, M., Colgan, A., Owen, S. V., Sivasankaran, S. K., et al. (2015). RNA-seq brings new insights to the intra-macrophage transcriptome of Salmonella Typhimurium. PLoS Pathog. 11:e1005262. doi: 10.1371/journal.ppat.1005262

Steele-Mortimer, O. (2008). The Salmonella-containing vacuole-moving with the times. Curr. Opin. Microbiol. 11, 38-45. doi: 10.1016/j.mib.2008.01.002

Strauss, E. (1999). Anti-immune trick unveiled in Salmonella. Science 285, 306-307. doi: 10.1126/science.285.5426.306b

Taylor, M., Cox, W., Otterstatter, M., de With, N., and Galanis, E. (2018). Evaluation of agricultural interventions on human and poultry-related Salmonella Enteritidis in British Columbia. Foodborne Pathog. Dis. 15, 39-43. doi: 10.1089/fpd.2017.2302

Tobar, J. A., Carreno, L. J., Bueno, S. M., Gonzalez, P. A., Mora, J. E., Quezada, S. A., et al. (2006). Virulent Salmonella enterica serovar typhimurium evades adaptive immunity by preventing dendritic cells from activating $\mathrm{T}$ cells. Infect. Immun. 74, 6438-6448. doi: 10.1128/IAI.00063-06

Uchiya, K., Barbieri, M. A., Funato, K., Shah, A. H., Stahl, P. D., and Groisman, E. A. (1999). A Salmonella virulence protein that inhibits cellular trafficking. EMBO J. 18, 3924-3933. doi: 10.1093/emboj/18.14.3924

Uchiya, K., Groisman, E. A., and Nikai, T. (2004). Involvement of Salmonella pathogenicity island 2 in the upregulation of interleukin-10 expression in macrophages: role of protein kinase A signal pathway. Infect. Immun. 72, 1964-1973. doi: 10.1128/IAI.72.4.1964-1973.2004

Uchiya, K., and Nikai, T. (2004). Salmonella enterica serovar Typhimurium infection induces cyclooxygenase 2 expression in macrophages: involvement of Salmonella pathogenicity island 2. Infect. Immun. 72, 6860-6869. doi: 10.1128/IAI.72.12.6860-6869.2004
Uchiya, K., and Nikai, T. (2005). Salmonella pathogenicity island 2-dependent expression of suppressor of cytokine signalling 3 in macrophages. Infect. Immun. 73, 5587-5594. doi: 10.1128/IAI.73.9.5587-5594.2005

Uchiya, K., and Nikai, T. (2008). Salmonella virulence factor SpiC is involved inexpression of flagellin protein and mediates activation of the signal transduction pathways in macrophages. Microbiology 154, 3491-3502. doi: 10.1099/mic.0.2008/021667-0

Uchiya, K., and Nikai, T. (2009). Involvement of SPI-2-encoded SpiC in flagellum synthesis in Salmonella enterica serovar Typhimurium. BMC Microbiol. 9 1-10. doi: 10.1186/1471-2180-9-179

Valdez, Y., Ferreira, R. B. R., and Finlay, B. B. (2009). Molecular mechanisms of Salmonella virulence and host resistance. Curr. Top. Microbiol. Immunol. 337, 93-127. doi: 10.1007/978-3-642-01846-6_4

Vazquez-Torres, A., Xu, Y., Jones-Carson, J., Holden, D. W., Lucia, S. M., Dinauer, M. C., et al. (2000). Salmonella pathogenicity island2-dependent evasion of the phagocyte NADPH oxidase. Science 87, 1655-1658. doi: 10.1126/science.287.5458.1655

Walthers, D., Carroll, R. K., Navarre, W. W., Libby, S. J., Fang, F. C., and Kenney, L. J. (2007). The response regulator SsrB activates expression of diverse Salmonella pathogenicity island 2 promoters and counters silencing by the nucleoid-associated protein H-NS. Mol. Microbiol. 65, 477-493. doi: 10.1111/j.1365-2958.2007.05800.x

Yu, X. J., Liu, M., and Holden, D. W. (2004). SsaM and SpiC interact and regulate secretion of Salmonella pathogenicity island 2 type III secretion system effectors and translocators. Microbiology 54, 604-619. doi: 10.1111/j. 1365-2958.2004.04297.x

Yu, X. J., McGourty, K., Liu, M., Unsworth, K. E., and Holden, D. W. (2010). pH sensing by intracellular Salmonella induces effector translocation. Science 328, 1040-1043. doi: 10.1126/science. 1189000

Yu, X. J., Ruiz-Albert, J., Unsworth, K. E., Garvis, S., Liu, M., and Holden, D. W. (2002). SpiC is required for secretion of Salmonella pathogenicity island 2 type III secretion system proteins. Cell. Microbiol. 4, 531-540. doi: 10.1046/j. 1462-5822.2002.00211.x

Zeng, L., Li, B., Yan, S., Pan, Z., Geng, S., and Jiao, X. (2015). Construction of genetically engineered attenuated vaccines Salmonella entertidis C50041 spiCAcrp. Chin. J. Zoonoses 31, 97-101. doi: 10.3969/cjz.j.issn.10022694.2015.02.001

Conflict of Interest Statement: The authors declare that the research was conducted in the absence of any commercial or financial relationships that could be construed as a potential conflict of interest.

Copyright (c) 2019 Wang, Cai, Zhang, Liu, Gong, Pan, Geng and Jiao. This is an open-access article distributed under the terms of the Creative Commons Attribution License (CC BY). The use, distribution or reproduction in other forums is permitted, provided the original author(s) and the copyright owner(s) are credited and that the original publication in this journal is cited, in accordance with accepted academic practice. No use, distribution or reproduction is permitted which does not comply with these terms. 